\title{
Follow-Up for Coping with Covid is as Important as Immunity Boosting- A Short Survey in Uttar Pradesh
}

\author{
Khushboo Rastogi ${ }^{1 *}$ and Shalini Agarwal ${ }^{2}$ \\ ${ }^{1}$ Professor and HOD, Department of Dentistry, Saraswathi Institute of Medical \\ Sciences, Uttar Pradesh, India \\ ${ }^{2}$ Department of Dietetics, Saraswathi Institute of Medical Sciences, Uttar Pradesh, \\ India \\ *Corresponding Author: Khushboo Rastogi, Professor and HOD, Department of \\ Dentistry, Saraswathi Institute of Medical Sciences, Uttar Pradesh, India.
}

Received: September 15, 2021

Published: October 13, 2021

(C) All rights are reserved by Khushboo

Rastogi and Shalini Agarwal.

\begin{abstract}
Coronavirus disease 2019 (COVID-19) has caused disturbance and fear in everyone's life. The importance of immunity booster is important but after this sudden pandemic occurrence it is very important to normalize routine step by step. Adequate intake of micro and macro nutrients, slow and steady physical exercise and adequate fluid-intake will gradually boost immunization. The purpose of this article is to provide guidance as how to take easy steps to normalize the life and routine after COVID-19.
\end{abstract}

Keywords: Covid-19; Immunity Booster; Physical Exercise; Dietary Changes

\section{Introduction}

Covid-19 has caused disruption in livelihood of entire world. It has not only affected health but also economy exponentially. Coronavirus disease is an infectious disease caused by a newly discovered coronavirus whose protein has matched with one of the types of SARS disease.

Most individuals infected with this virus experiences mild to moderate respiratory illness and recovers without requiring special treatment. Older people and patients with co-morbid conditions like CVS, diabetes, chronic respiratory disease and cancer are more likely to develop serious debilitating condition.

The best way to prevent and slow down transmission is to be well informed about the covid-19 virus, the disease it causes and how it spreads [1]. If you have just recovered from covid-19 or re- turned home from the hospital, you have already won the war. The major battle has been overcome but one has to be readied for winning minor battles in order to achieve normal routine [2].

A survey was done of approximately 100 patients who were admitted in Saraswathi Institute of Medical Sciences, Hapur as covid-19 positive and Performa were asked to be duly filled by patients after one month and 3 months of discharge exclusively from Uttar Pradesh. The Performa consisted of following questionnaire:

1. Low energy levels and fatigue.

2. Difficulty in breathing/chest congestion/cough.

3. Loss of appetite.

4. Anxiety and fear.

5. Diet Modification as advised during isolation, after discharge and follow up. 


\section{Material and Method}

Total 100 patients aged 25 - 40 yrs were included who had mild to moderate symptoms of covid-19. Patients who were admitted in ICU at high risk or underlying co-morbidity were excluded from the study due to prolongation in recovery and severity of disease. The aim was to determine successful recovery after covid-19 step by step.

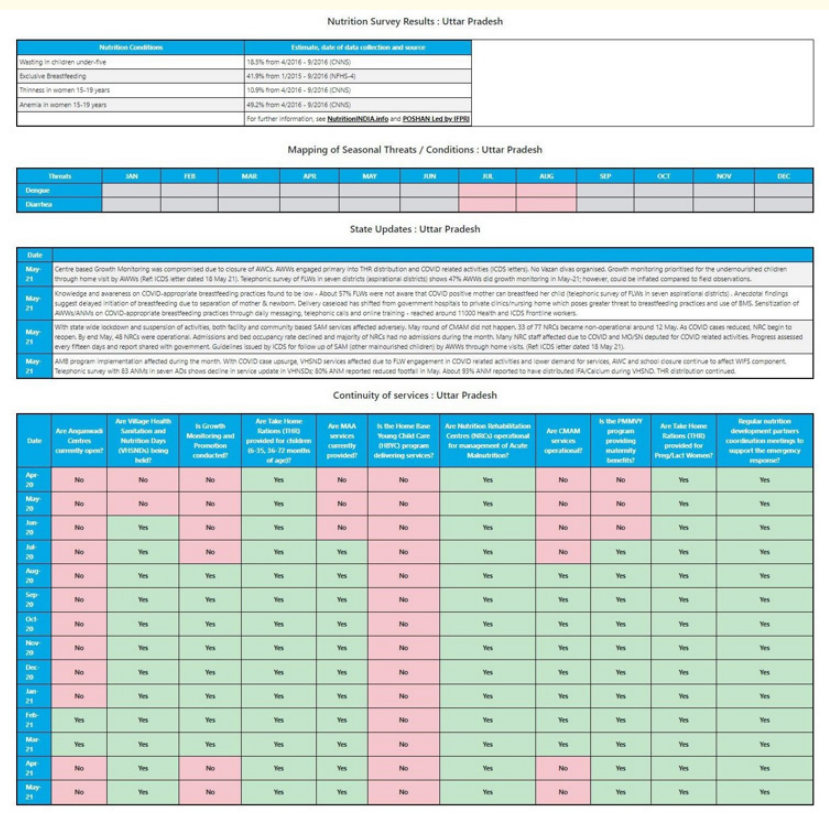

Figure 1

\section{Discussion}

Covid-19 is a deadly virus which has not only ceased our daily priorities but also has affected our financial and mental well-being. It has made us question the importance of a healthy lifestyle. The role of immunity in coping with this disease has amicably led to the belief of taking sufficient time for ourselves from our busy schedule. Following survey was conducted to clearly understand the effects of covid-19 virus on physical and mental well being, role of immunity and guidance to cope from it.

\section{Low energy levels and fatigue}

Out of 100 patients included $75 \%$ of covid patients suffered from low energy levels and fatigue even after 1 month of discharge which subsequently was observed less after 12 weeks/3 months of survey. The possible explanation was lack of muscle/physical mobility. After only a day or two in bed, significant loss of muscle mass is evident and regaining that loss takes time [3]. Sports medicine doctors and researchers have recommended to take it slow and gradually reintroduce physical activity to their routine followed with recommended diet modification which included introduction of Omega3 fatty acid and spirulina for gradual recovery [4].

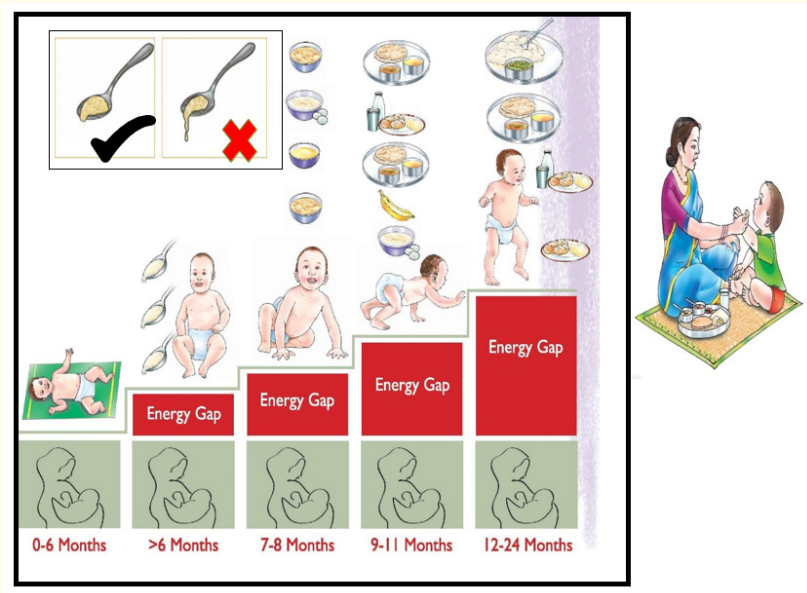

Figure 2

\section{Difficulty in breathing/chest congestion and cough}

Follow up is the key factor to overcome from the ill effects caused by this virus to lungs leading to abnormalities. Computed Tomography scans of the chest, echocardiography, lung function testing, clinical examinations and laboratory analysis at regular intervals are key factors to determine effective recovery from this deadly virus. About $88 \%$ of the people studied showed structural lung abnormalities on the CT scans of 1 month after discharge which subsequently reduced to $56 \%$ after scans of 3 months of discharge. Those who had respiratory symptoms like pneumonia were recommended to rest for at least a week after symptoms subside followed by gradual returning to physical activity with an emphasis laid on monitoring their breathing [5].

\section{Loss of appetite}

Approximately in more than $60 \%$ of patients loss of appetite and gastrointestinal symptoms were observed which subsided 
to $45 \%$ after a month and $30 \%$ after 3 months of follow up. They were advised to keep tabs on their fluid and calorie intake while easing into their fitness patterns. They were advised to introduce ayurvedic kada, spirulina and omega 3 fatty acid as regular regime for gradual recovery [6].

\section{Fear and anxiety}

Social distancing and isolation has further added to increased levels of stress and suicidal tendencies in adults as well as in children. Therefore, it is very important to keep a check on the mental status of an individual [7]. Out of 100 patients who participated in the survey almost all were suffering from increased stress levels and fear of inability to recover from this disease. Regular sessions with doctor, good sleep and eating healthy has led to significant decrease in levels of fear and anxiety thereby leading to speedy mental recovery [8].

Diet modification

Diet modification has led to positive effects on effective recovery against covid-19 [9]. Introduction of balanced diet with immunity boosters in the form of spirulina, omega 3 fatty acids and increase intake of fluids have led to speedy recovery among patients after discharge [10].

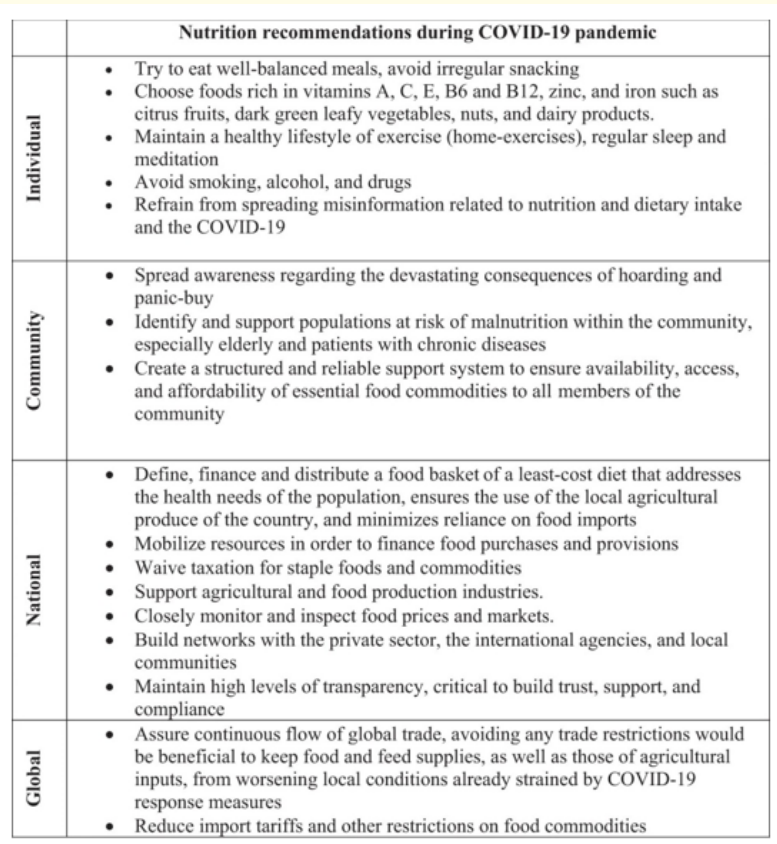

Figure 3

\section{Conclusion}

Follow up is as important as immunity boosting in coping up with the symptoms of covid -19 for speedy and effective recovery.
It is recommended to get advice of your physician and team of specialists who helped you during covid -19 to formulate an effective dietary and exercise plan to effectively cope up with ill effects of covid-19.

\section{Conflict of Interest}

Authors have no conflict of interest among them.

\section{Bibliography}

1. WHO international Spread of Covid-19: General Information.

2. Van den Borst B., et al. "Comprehensive health assessment three months after recovery from acute covid-19". Clinical Infectious Diseases (2020): 21.

3. Korin Miller. "Is fatigue a sign of Covid-19? What yoy need to know, according to experts" (2020).

4. Krunal Pandav and Rafael Abreu. "Omega 3 fatty acids and covid-19: A comprehensive review: Donald Hathaway, III". Infect Chemother 52.4 (2020): 478-495.

5. Faverio P., et al. "Six-month Pulmonary after Covid-19- A prospective, Multicentre follow-up study.

6. Saray Gutiérrez., et al. "Effects of omega-3 fatty acids on immune cells". International Journal of Molecular Sciences 20 (2019): 5028.

7. Li LZ and Wang S. "Prevalence and predictors of general psychiatric disorders and loneliness during covid-19 in the UK". Psychiatry Research 291 (2020): 113267.

8. Emily A Holmes., et al. "Multidisciplinary Research priorities for the covid-19 pandemic: a call for action for mental health science". Lancet Psychiatry 7 (2020): 547-560.

9. Sakshi Chopra., et al. "Impact of Covid-19 on lifestyle related behaviours- a cross- sectional audit of responses from nine hundred and ninety five participants from India". Diabetes and Metabolic Syndrome 14.6 (2020): 2021-2030.

10. Jain Swati and Ranjan Shobhana. "Evidence that maintaining optimal nutrition status for a well-functioning immune system might promote recovery for mild covid-19 patients". World $\mathrm{Nu}$ trition 11.4 (2020): 66-93.

Volume 5 Issue 11 November 2021 (C) All rights are reserved by Khushboo Rastogi and Shalini Agarwal. 